\title{
ANÁlISE DE AGRUPAMENTO, COM BASE NA COMPOSIÇÃo FÍsICO-gUíMiCA, DE AMOSTRAS DE MÉIS PRODUZIDOS POR Apis mellifera $L$. NO ESTADO DE SÃO PAULO ${ }^{1}$
}

\author{
Luís Carlos MARCHINI ${ }^{2, *}$, Augusta Carolina de Camargo Carmello MORETI ${ }^{3}$, Ivani Pozar OTSUK ${ }^{4}$
}

\begin{abstract}
RESUMO
A composição do mel depende, basicamente, da composição do néctar de cada espécie vegetal produtora, conferindo-lhe características específicas enquanto que as condições climáticas e o manejo do apicultor têm influência menor sobre essas características. A presente pesquisa, desenvolvida com amostras de méis de Apis mellifera coletadas diretamente dos produtores de 84 municípios do Estado de São Paulo teve o objetivo de verificar, com base em características físico-químicas, como se agrupam as amostras de méis silvestres e de eucaliptos. Dentre as 121 amostras de méis analisadas as de eucaliptos e as silvestres formam grupos distintos quanto aos caracteres físico-químicos, o que confirma que a origem floral interfere decisivamente nas características dos méis. Pela análise dos componentes principais, pode-se verificar que os caracteres que mais influenciaram no agrupamentos das amostras de méis foram condutividade elétrica e quantidade de $\mathrm{K}$, no eixo $\mathrm{X}$ e índice de formol e umidade, no eixo $\mathrm{Y}$.

Palavras-chave: análise de agrupamento; Apis mellifera; composição do mel.
\end{abstract}

\section{SUMMARY}

CLUSTER ANALYSIS, WITH BASIS IN PHYSICO-CHEMICAL COMPOSITION, OF SAMPLES OF HONEY PRODUCED BY Apis mellifera L. IN SÃO PAULO STATE. The honey composition depends, basically, of the nectar composition of each vegetal species. This composition confer to it specific characteristics while that the climatic conditions and the beekeeper handling have lesser influence on these features. The present research, developed with samples of Apis mellifera honeys, collected directly of the producers from 84 locations of the State of São Paulo, Brazil, had the objective of verifying, on the basis of physico-chemical characteristics, as the samples of wild and Eucalyptus honeys are grouped. Amongst the 121 samples analyzed the wild and Eucalyptus honeys form distinct groups with basis of the physico-chemical characteristics, what confirms that the floral origin intervenes decisively with the honey characteristics. For the analysis of the main components, it can be verified that the characters that had more influenced in the groupings of the honey samples were the electric conductivity and amount of $\mathrm{K}$, in axle $\mathrm{X}$ and the formol index and humidity, in axle $\mathrm{Y}$.

Keywords: Apis mellifera; honey composition; cluster analysis.

\section{1 - INTRODUÇÃO}

Como o mel é resultado da desidratação e transformação do néctar, a quantidade de mel que pode ser obtida de uma determinada planta varia com os fatores que influenciam a produção e a concentração de néctar e, ainda, com a concentração e proporções de seus carboidratos, com a quantidade de flores da área e com o número de dias em que as flores estão secretando néctar [25]. A composição do mel depende, basicamente, da composição do néctar de cada espécie vegetal produtora, conferindo-lhe características específicas enquanto que as condições climáticas e o manejo do apicultor têm influência menor [65].

Existe no Brasil uma legislação específica para mel a qual estabelece parâmetros de controle de qualidade para o produto, com indicação das análises e métodos a serem empregados [12].

Dentre os açúcares existentes no mel, os monossacarídeos constituem a maior parte, variando de $85 \%$

1. Recebido para publicação em 16/01/2002. Aceito para publicação em 02/02/2005 (000795). Parte da tese de Livre Docência apresentada pelo primeiro autor à ESALQ/USP.

2. Departamento de Entomologia, Fitopatologia e Zoologia Agicola, ESALQ/ USP, Piracicaba, SP

3. Centro de Pesquisa e Desenvolvimento de Zootecnia Diversificada do Instituto de Zootecnia/APTA/SAA, Nova Odessa, SP.

4. Centro de Pesquisa e Desenvolvimento de Genética e Reprodução Animal do Instituto de Zootecnia/ APTA/SAA, Nova Odessa, SP.

* A quem a correspondência deve ser enviada. a 95\% da sua composição [33, 50, 63]. Outro parâmetro importante é a umidade que pode influenciar na viscosidade, no peso específico, maturidade, cristalização, sabor, conservação e palatabilidade [50, 65]. Com a descristalização do mel pode se formar o hidroximetilfurfural (HMF) o qual aumenta com a elevação da temperatura, com armazenamento do mel, adição de açúcar invertido, sendo também afetado pela acidez, $\mathrm{pH}$, água e minerais $[22,30,49,50,59,62]$. Dentre as enzimas, a diastase é a principal, sendo um parâmetro importante na avaliação da qualidade do mel [11, 57].

Os méis brasileiros possuem uma variação grande de cor o que pode influenciar na preferência do consumidor, que, na maioria das vezes, escolhe o produto apenas pela aparência. Tal é a relevância deste parâmetro que o INTERNATIONAL TRADE FORUM [36] considerou a cor como uma das características do mel que tem particular importância no mercado internacional. A cor do mel está correlacionada com a sua origem floral, o processamento e armazenamento, fatores climáticos durante o fluxo do néctar, a temperatura na qual o mel amadurece na colméia [50] e o conteúdo de minerais presentes, que muitas vezes são expressos como cinzas [8, 10, 39, 45, 58].

A condutividade elétrica pode ser utilizada como método suplementar na determinação da origem botânica do mel [1] e tem correlação com o conteúdo de cinzas, pH, acidez, sais minerais, além das proteínas e outras substâncias presentes no mel [10, 25, 55]. 
Apesar do pouco conhecimento sobre as características do material protéico, a ocorrência de proteínas em mel é utilizada na detecção de adulteração do produto comercial [26].

Outro parâmetro importante para comprovar a autenticidade do mel é o índice de formol que pode indicar a presença de produtos artificiais, quando seu valor é baixo ou a presença de hidrolizado de proteínas, quando seu valor é excessivamente alto [52].

O conhecimento da origem floral dos méis é muito importante para a caracterização do produto. Assim, a análise polínica do mel é um importante instrumento para o reconhecimento das plantas apícolas utilizadas pelas abelhas, como suprimento de néctar e pólen [7, 34, 37].

A presente pesquisa, desenvolvida com amostras de méis de Apis mellifera de 84 municípios do Estado de São Paulo teve o objetivo de verificar, com base em características físico-químicas, como se agrupam as amostras de méis silvestres e de eucaliptos.

\section{2 - MATERIAL E MÉTODOS}

As amostras de méis (num total de 121) foram obtidas através de contato direto com apicultores de diferentes localidades do Estado de São Paulo, conforme Tabela 1. As amostras foram coletadas em diferentes épocas do ano dependendo da origem floral (silvestre ou eucalipto) e acondicionadas em frascos de vidro, devidamente etiquetados com as informações do local de produção e transportados para o laboratório.

As análises físico-químicas e polínicas dos méis produzidos por Apis mellifera foram realizadas no Laboratório de Apicultura do Departamento de Entomologia, Fitopatologia e Zoologia Agrícola da Escola Superior de Agricultura "Luiz de Queiroz", campus de Piracicaba, da Universidade de São Paulo.

Todos os parâmetros analisados são resultantes da média de três repetições.

\section{1 - Análises físico-químicas dos méis}

\subsection{1 - Umidade}

A umidade das diferentes amostras de méis foi determinada por meio de um refratômetro manual ATAGO (luz natural, temperatura ambiente) específico para mel [4]. Este aparelho foi adaptado a partir do refratômetro Abbé e possui um alto contraste no campo de visão [12].

\subsection{2 - Cor}

Para a verificação da cor do mel foi utilizado o colorímetro fotoelétrico DME-11 com comprimento de onda de $520 \mathrm{~mm}$, em célula de $1 \mathrm{~cm}$.

Por meio de comparação dos dados obtidos com os da tabela de Townsend, citado por PAMPLONA [46], determinou-se a cor do mel.
TABELA 1. Número de amostras de méis de flores silvestres (S) e de eucaliptos (E) coletadas em diferentes municípios do Estado de São Paulo.

\begin{tabular}{|c|c|c|c|c|c|}
\hline \multirow{2}{*}{$\begin{array}{l}\text { Municípios do } \\
\text { Estado de São Paulo }\end{array}$} & \multicolumn{2}{|c|}{ Méis } & \multirow{2}{*}{$\begin{array}{l}\text { Municípios do } \\
\text { Estado de São Paulo }\end{array}$} & \multicolumn{2}{|c|}{ Méis } \\
\hline & S & $E$ & & $\mathrm{~S}$ & $E$ \\
\hline Altinópolis & 1 & 1 & Jundiaí & 3 & \\
\hline Amparo & & 1 & Junqueirópolis & 2 & \\
\hline Andradina & 1 & & Juquitiba & 1 & \\
\hline Anhembi & & 1 & Lagoinha & 1 & \\
\hline Araçatuba & 2 & 1 & Lençóis Paulistas & 1 & 2 \\
\hline Araraquara & & 1 & Lins & 1 & \\
\hline Assis & 1 & & Luiz Antonio & & 2 \\
\hline Auriflama & 2 & & Mirandópolis & 1 & \\
\hline Avaré & & 2 & Mirassol & 1 & \\
\hline Bananal & 1 & & Monte Alegre do Sul & 1 & \\
\hline Barretos & 1 & 1 & Monteiro Loubato & 1 & 1 \\
\hline Bebedouro & & 1 & Oriente & 1 & \\
\hline Boa Esperança do Sul & 1 & & Paraibuna & 1 & \\
\hline Bofete & 1 & & Paranapanema & 1 & \\
\hline Botucatu & & 1 & Piracaia & 1 & \\
\hline Brotas & 1 & 2 & Piracicaba & 1 & \\
\hline Caçapava & 1 & 1 & Piracuama & 1 & \\
\hline Capão Bonito & 1 & & Pirassununga & 1 & \\
\hline Capela do Alto & 1 & & Presidente Prudente & 1 & \\
\hline Castilho & 1 & & Redenção da Serra & 2 & \\
\hline Colina & 1 & & Rio Claro & 1 & 1 \\
\hline Corumbatai & 1 & & Sabino & 2 & \\
\hline Cunha & 1 & & Santa Cruz do Rio Pardo & 1 & \\
\hline Espírito Santo do Pinhal & 1 & & Santa Fé do Sul & 1 & \\
\hline Estrela d'Oeste & 2 & & Santo Antonio do Pinhal & 1 & \\
\hline Gália & 1 & & São Carlos & 1 & \\
\hline Guarani d'Oeste & 1 & & São João da Boa Vista & 1 & \\
\hline Guareí & 2 & & São José do Rio Preto & 2 & 1 \\
\hline Guarujá & 1 & & São Luis do Paraitinga & 2 & \\
\hline Igarapava & 1 & & São Pedro & 2 & 1 \\
\hline Ilha Solteira & 1 & & São Roque & 3 & \\
\hline Ipeúna & & 1 & Serra Negra & 5 & \\
\hline Itaberá & 1 & & Sete Barras & 1 & \\
\hline Itanhaém & 1 & & Taubaté & 1 & \\
\hline Itapetininga & 1 & 1 & Tietê & 1 & \\
\hline Itapeva & 1 & & Torrinha & 2 & 1 \\
\hline Itapira & 1 & 1 & Tremembé & 1 & \\
\hline Itapirapuã Paulista & 1 & & Ubatuba & 1 & \\
\hline Ituverava & 1 & & Vargem Grande do Sul & 1 & 1 \\
\hline Jaboticabal & & 1 & Valentin Gentil & 1 & \\
\hline Jarinu & 1 & & Vista Alegre do Alto & 1 & \\
\hline Joanópolis & 1 & & Votorantin & 1 & \\
\hline
\end{tabular}

Nota: Somente após a análise polínica dos méis obtidos foram realizadas as análises físico-químicas.

\subsection{3 - Minerais}

Os diferentes minerais contidos no mel foram determinados a partir das cinzas das amostras dos méis, 
em solução de $\mathrm{HCl}$ a 50\%, por espectrofotometria de emissão em chama e sistemas de injeção em fluxo contínuo conforme Bergamim Filho et al. citado por PAMPLONA [46], com espectofotômetro Micronal, modelo B262; Bomba Peristáltico modelo B332 e sistemas de injeção em fluxo contínuo: injetor computador e microcomputador SACI. Foram determinados os seguintes minerais: $\mathrm{P}, \mathrm{K}, \mathrm{Ca}, \mathrm{Mg}, \mathrm{S}, \mathrm{Cu}, \mathrm{Fe}, \mathrm{Mn}, \mathrm{Zn}$ e Na.

\subsection{4 - Condutividade elétrica}

A condutividade elétrica foi obtida em uma solução a $20 \%$ de matéria seca de mel a $20^{\circ} \mathrm{C}$ [9]. Para tanto foi utilizado o condutivímetro Hanna, modelo HI 8820, para obtenção dos dados.

\subsection{5 - Proteínas}

As proteínas do mel foram determinadas seguindose o método micro-Kjeldahl citado nas normas analíticas do Instituto Adolfo Lutz [48], utilizando-se, para o cálculo do valor de proteínas, o fator 6,25.

\subsection{6 - Teor de cinzas}

A determinação de cinzas foi realizada conforme a metodologia citada na legislação brasileira por em mufla a $550^{\circ} \mathrm{C}$ até peso constante BRASIL [12].

\subsection{7 - pH, acidez e indice de formol}

$\mathrm{O} \mathrm{pH}$, a acidez e o índice de formol foram determinados segundo a metodologia de MORAES \& TEIXEIRA [43].

\subsection{8 - Diastase}

$\mathrm{O}$ índice de diastase foi determinado conforme a metodologia citada na legislação brasileira [12].

\subsection{9 - Hidroximetilfurfural}

O hidroximetilfurfural foi determinado conforme a metodologia citada na legislação brasileira [12].

\subsubsection{0 - Açúcares totais, açúcares redutores e} sacarose aparente

A determinação de açúcares totais, açúcares redutores e sacarose aparente foi realizada por meio do método estabelecido pela COPERSUCAR [21]

\section{2 - Análise polínica}

Todas as amostras foram submetidas ao método da acetólise e em seguida submetidas a uma análise qualitativa e outra quantitativa [28]. Somente após a análise polínica dos méis obtidos foram realizadas as análises físico-químicas.

\section{3 - Análise dos dados}

Os dados foram analisados por meio da análise multivariada, utilizando-se a análise de componentes principais para avaliar a importância de cada caracter físicoquímico estudado sobre a variação total disponivel [40].
Esta técnica baseia-se na padronização e rotação dos eixos ortogonais (carácter físico-químico), gerando um novo conjunto de coordenadas (componentes principais) não correlacionados entre si [44]. Foi realizado um descarte dos caracteres altamente correlacionados (condutividade elétrica, $\mathrm{pH}, \mathrm{K}$ e $\mathrm{Mg}$ ) e utilizando-se o critério proposto por JOLIFFE [38], desprezando-se a variável de maior coeficiente em cada componente com autovalor menor que 0,70.

Para a análise de agrupamento adotou-se segundo BUSSAB, ANDRADE \& MYAZAKY [13], como média de dissimilaridade a distância euclidiana média para os dados devidamente padronizados. Os agrupamentos foram formados pelo método UPGA (unweighted pairgroup average).

\section{3 - RESULTADOS E DISCUSSÃO}

\section{1 - Análises físico-químicas}

\subsection{1 - Açúcares redutores, totais e sacarose aparente}

Os valores médios obtidos (Tabela 2) em porcentagem de açúcares redutores, açúcares totais e sacarose aparente para méis de eucalipto foram: 72,30 (67,70 a $77,10), 74,90(67,80$ a 88,30$)$ e $2,40(0,10$ a 15,20), respectivamente. Já para os méis silvestres os valores médios para açúcares redutores, açúcares totais e sacarose aparente, foram de: 72,60 (53,20 a 80,00); 75,20 $(68,20$ a 82,00$) ; 2,40(0,20$ a 27,40$)$, respectivamente. Esses valores são próximos aos obtidos por CAMPOS [14], SODRÉ [53] e VILHENA \& ALMEIDA-MURADIAN [60], estudando méis brasileiros, e aos de VIT-OLIVIER [61]; SPORNS, PLHAK \& FRIEDRICH [54] e BALDI-CORONEL, DALL'OGLLIO \& LEZCANO [6] utilizando méis de outros países.

CANO et al. [17] verificaram que 6 (10,3\%) das 173 amostras de méis comecializados em São Paulo, capital, continham valores de sacarose aparente acima dos permitidos pela legislação vigente no BRASIL [12].

Os valores médios observados para açúcares redutores e sacarose aparente, encontram-se dentro dos limites estabelecidos pela legislação brasileira [12].

\subsection{2 - Umidade}

A legislação brasileira estabelece que a umidade do mel não deve exceder $20 \%$ [12]. No presente trabalho, embora as médias observadas para méis silvestres tenha sido de $19,10 \%$ e para méis de eucalipto 21,20\% (Tabela 2), das 121 amostras de diferentes municípios do Estado de São Paulo, 44 amostras (36,40\%) apresentaram umidade superior a $20 \%$. Uma porcentagem considerável das amostras de méis está, portanto, fora das normas de qualidade do mel brasileiro.

CORNEJO \& TOMASEVICH [23] analisaram 12 amostras de méis de diferentes localidades dos Estados do Rio Grande do Sul e Santa Catarina, e encontraram 5 
amostras (41,67\%) com umidade superior a 20\%, enquanto CANO et al. [17] observaram 14 amostras (24,1\%) acima dos valores permitidos pela legislação, em 173 amostras analisadas de méis consumidos em São Paulo, capital. GAMERO, CORNEJO \& TOMASEVICH [31] analisaram 169 amostras de méis de diferentes localidades na Argentina, e apenas 12 amostras apresentaram umidade superior a $20 \%$.

CANO et al. [16] comparando amostras de méis brasileiros de flores de eucalipto e de laranjeira, verificaram que a umidade variou de 16,9 a $18,4 \%$ para eucalipto e 15,5 a $16,8 \%$ para os de flores de laranjeira, valores próximos aos obtidos por VILHENA \& ALMEIDAMURADIAN [60] e aos do presente trabalho.

A explicação para o elevado teor de água encontrado neste experimento, principalmente nas amostras de méis de eucaliptos, poderia ser a colheita do mel oriundo de favos não operculados ou o período de armazenamento, podendo assim, o mel ter absorvido umidade do ambiente.

\subsection{3 - Condutividade elétrica}

Para os valores de condutividade elétrica, observouse grande variação entre as amostras analisadas (Tabela 2). A média de $1018,65 \mu \mathrm{S} \mathrm{cm}^{1}(331,00$ a 1257,33$) \mathrm{de}$ condutividade elétrica para as amostras de méis de eucaliptos das diferentes localidades do Estado de São Paulo, pode ser considerada elevada, comparando com o valor médio de $448,60 \mu \mathrm{S} \mathrm{cm}^{-1}$, encontrado por GOMEZ et al. [32], para amostras de méis comerciais de eucaliptos da Espanha. Já para méis silvestres foi observada uma média de $568,25 \mu \mathrm{S} \mathrm{cm}^{-1}(160,71$ a 1251,60).

SODRÉ [53] obteve valores de condutividade elétrica para méis da região litoral norte do Estado da Bahia variando de 271,67 a 1634,00 $\mu \mathrm{S} . \mathrm{cm}^{-1}$, com uma média de $780,70 \mu \mathrm{S} . \mathrm{cm}^{-1}$.

\subsection{4 - Proteinas}

As porcentagens médias de proteínas encontradas foram de 0,32\% para méis de eucaliptos e 0,19\% para

TABELA 2. Médias, desvios padrão e intervalos de variação do índice de diastase, HMF, açúcares, proteínas, umidade, acidez, $\mathrm{pH}$, índice de formol, cinzas, minerais e condutividade elétrica de amostras de méis de diferentes municípios do Estado de São Paulo.

\begin{tabular}{|c|c|c|c|c|c|}
\hline \multirow[b]{2}{*}{ Caracteres } & \multirow[b]{2}{*}{ Normas vigentes [12] } & \multicolumn{2}{|c|}{ Mel silvestre } & \multicolumn{2}{|c|}{ Mel de Eucalipto } \\
\hline & & Médias $\pm \mathrm{s}(\mathrm{m})$ & $\begin{array}{l}\text { Intervalo de } \\
\text { variação }\end{array}$ & Médias $\pm \mathrm{s}(\mathrm{m})$ & $\begin{array}{l}\text { Intervalo de } \\
\text { variação }\end{array}$ \\
\hline Açúcares redutores (\%) & Mínimo 65 & $72,60 \pm 0,38$ & 53,20 a 80,00 & $72,30 \pm 0,47$ & 67,70 a 77,10 \\
\hline Açúcares totais (\%) & 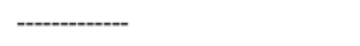 & $75,20 \pm 0,29$ & 68,20 a 82,00 & $74,90 \pm 0,69$ & 67,80 a 88,30 \\
\hline Sacarose aparente(\%) & Máximo 6 & $2,40 \pm 0,33$ & 0,20 a 27,40 & $2,40 \pm 0,56$ & 0,10 a 15,20 \\
\hline Umidade (\%) & Máximo 20 & $19,10 \pm 1,45$ & 16,00 a 23,40 & $21,20 \pm 1,23$ & 19,30 a 22,40 \\
\hline Condutividade elétrica $\left(\mu \mathrm{S} . \mathrm{cm}^{-1}\right)$ & (2) & $568,25 \pm 278,07$ & 160,71 a 1251,67 & $1018,65 \pm 471,64$ & 331,00 a 1257,33 \\
\hline Proteínas (\%) & 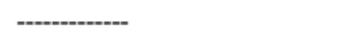 & $0,19 \pm 0,10$ & 0,05 a 0,58 & $0,32 \pm 0,08$ & 0,23 a 0,49 \\
\hline Cinzas (\%) & Máximo 0,60 & $0,25 \pm 0,16$ & 0,03 a 0,92 & $0,16 \pm 0,07$ & 0,07 a 0,32 \\
\hline Acidez (meq/kg) & Máximo 50 & $30,10 \pm 1,29$ & 14,00 a 75,50 & $33,80 \pm 2,22$ & 12,50 a 55,00 \\
\hline $\mathrm{pH}$ & 3,30 a 4,60 & $3,20 \pm 0,04$ & 2,30 a 5,00 & $3,60 \pm 0,08$ & 2,90 a 5,10 \\
\hline Índice de formol (mL/kg) & מ-m & $10,10 \pm 0,33$ & 5,00 a 20,50 & $6,90 \pm 0,31$ & 5,00 a 12,50 \\
\hline $\mathrm{HMF}(\mathrm{mg} / \mathrm{kg})$ & Máximo 60 & $19,30 \pm 1,69$ & 1,00 a 122,00 & $17,40 \pm 7,42$ & 0,30 a 207,20 \\
\hline Índice de diastase & $\begin{array}{l}\text { Número mínimo de } 8 \\
\text { (Gothe) ou } 3 \text { se o HMF }<15\end{array}$ & $17,30 \pm 0,93$ & 5,00 a 38,50 & $15,80 \pm 1,08$ & 5,00 a 23,80 \\
\hline \multicolumn{6}{|l|}{ Minerais: $(\mathrm{mg} / \mathrm{kg})$} \\
\hline $\mathrm{P}$ & & $16,65 \pm 19,39$ & 1,91 a 85,58 & $35,82 \pm 28,79$ & 8,00 a 155,00 \\
\hline $\mathrm{K}$ & & $990,85 \pm 646,61$ & 95,00 a 3140,00 & $1720,00 \pm 688,80$ & 562,00 a 3495,00 \\
\hline $\mathrm{Ca}$ & & $46,18 \pm 41,27$ & 1,00 a 202,00 & $187,70 \pm 68,16$ & 55,00 a 301,00 \\
\hline $\mathrm{Mg}$ & & $65,45 \pm 54,96$ & 11,00 a 286,00 & $128,74 \pm 63,51$ & 31,00 a 270,00 \\
\hline S & & $62,47 \pm 83,01$ & 1,00 a 500,00 & $51,82 \pm 24,07$ & 36,00 a 164,00 \\
\hline $\mathrm{Cu}$ & & $0,32 \pm 0,24$ & 0,10 a 2,00 & $0,34 \pm 0,12$ & 0,20 a 0,60 \\
\hline $\mathrm{Fe}$ & & $10,62 \pm 5,39$ & 3,90 a 44,70 & $5,35 \pm 1,78$ & 2,10 a 9,00 \\
\hline $\mathrm{Mn}$ & & $4,46 \pm 4,89$ & 0,20 a 23,30 & $8,43 \pm 4,36$ & 1,60 a 16,90 \\
\hline Zn & & $5,45 \pm 7,27$ & 1,20 a 48,30 & $2,65 \pm 1,69$ & 0,80 a 8,70 \\
\hline $\mathrm{Na}$ & & $49,31 \pm 26,36$ & 14,95 a 202,40 & $71,98 \pm 44,25$ & 23,70 a 212,50 \\
\hline
\end{tabular}

média \pm desvio padrão 
méis silvestres, com uma variação de 0,23\% a 0,49\% e 0,05 a 0,58 , respectivamente (Tabela 2 ).

Os valores de proteínas não diferiram muito dos obtidos por CIRILLI, PAPAGHEORGHEU \& SAVIGNI [20] e CAMPUS, MADAU \& SOLINAS [15], que observaram porcentagens de $0,20 \%$ e $0,60 \%$, respectivamente, para diferentes amostras de méis italianos.

AMARAL et al. [2] estudaram 9 amostras de méis de eucaliptos de diferentes regiões do Estado de São Paulo, e encontraram a média de $2,54 \%$ de proteínas, sendo essa média, bem superior à obtida no presente experimento.

IMPERATRIZ-FONSECA et al. [35], ao estudarem o teor de proteínas de amostras de méis de eucaliptos brasileiros, verificaram valores médios de 1,92 a 2,76\%. Essa porcentagem é bem maior que a média de 0,32\% verificada nas amostras de méis estudadas.

\subsection{5 - Cinzas}

A porcentagem de cinzas presente no mel expressa a riqueza do material mineral, e se constitui em parâmetro bastante utilizado nas determinações que visam verificar a qualidade do mel.

A legislação brasileira estabeleceu que o máximo de cinzas presentes nos méis deve ser de 0,60\% [12]. Baseando-se, portanto, na legislação, pode-se verificar que as amostras de méis de eucaliptos e silvestres, apresentaram porcentagens médias de cinzas abaixo do máximo permitido, estando, portanto, dentro das normas para méis de boa qualidade (Tabela 2).

A porcentagem média de cinzas das amostras de méis de eucaliptos analisadas foi de $0,16 \%$, valor esse um pouco inferior aos $0,20 \%$ de amostras de méis comerciais de eucaliptos, encontradas na Espanha; enquanto para os méis silvestres a média obtida foi de 0,25\% [32].

MORAES \& MANTOVANI [42] ao estudarem o teor de cinzas de amostras de méis brasileiros de cana, carrapicho e eucalipto encontraram teor de 1,20\% de cinzas, valor esse bastante superior ao das amostras de méis analisadas no presente experimento.

Os valores de cinzas encontrados neste experimento (Tabela 2) foram semelhantes aos obtidos por FLECHTMANN et al. [29] em amostras de méis de eucaliptos de diferentes regiões do Estado de São Paulo e próximos à média obtida por VILHENA \& ALMEIDAMURADIAN [60] que foi de $0,14 \%$, também para méis deste Estado.

\subsection{6 - pH, acidez e indice de formol}

Os valores de $\mathrm{pH}$ de $3,60(2,90$ a 5,10$)$ e $3,20(2,30$ a 5,00 ), respectivamente, para méis de eucaliptos e silvestres (Tabela 2), estão próximos aos obtidos por AZEREDO \& AZEREDO [5]; FLECHTMANN et al. [29]; HORN et al. [33]; PAMPLONA [46] e SODRÉ [53] para méis brasileiros; aos de BALDI-CORONEL, DALL'OGLLIO \& LEZCANO [6] para méis argentinos e aos de ANDRADE et al. [3] em méis portugueses.
Para a acidez, os valores médios obtidos foram $33,80 \mathrm{meq} / \mathrm{kg}(12,50$ a $55,00 \mathrm{meq} / \mathrm{kg})$ para méis de eucaliptos e $30,10 \mathrm{meq} / \mathrm{kg}(14,00$ a $75,50 \mathrm{meq} / \mathrm{kg})$ para méis silvestres. Os valores encontrados estão próximos aos de PAMPLONA [46], CARVALHO et al. [18], SODRÉ [53] e superiores à média obtida por VILHENA \& ALMEIDAMURADIAN [60].

CANO et al. [17] observaram que 21 (36,2\%) das 173 amostras de méis comercializados na cidade de São Paulo apresentavam valores de acidez acima dos permitidos enquanto no presente trabalho os valores foram de $19,1 \%$ para méis silvestres e $29,6 \%$ para méis de eucaliptos.

Os valores de índice de formol encontrados para méis de eucaliptos variaram de 5,00 a $12,50 \mathrm{~mL} / \mathrm{kg}$ com uma média de $6,90 \mathrm{~mL} / \mathrm{kg}$ e de 5,00 a $20,50 \mathrm{~mL} / \mathrm{kg}$ com uma média de $10,10 \mathrm{~mL} / \mathrm{kg}$ para méis silvestres.

Os valores observados estão próximos aos obtidos por TEMIZ [56], CARVALHO et al. [19] e SODRÉ [53].

\subsection{7 - Hidroximetilfurfural}

As quantidades médias de hidroximetilfurfural (HMF) encontradas nas amostras de méis analisadas variaram, para méis de eucaliptos de $17,40 \mathrm{mg} / \mathrm{kg}(0,30$ a $207,20 \mathrm{mg} / \mathrm{kg}$ ) e de $19,30 \mathrm{mg} / \mathrm{kg}(1,00$ a $122,00 \mathrm{mg} / \mathrm{kg})$ para méis silvestres (Tabela 2). O limite estabelecido pela legislação brasileira [12] para HMF é de 60mg/kg, e desta forma sete amostras $(5,8 \%)$ estão fora destas especificações.

DAYRELL \& VITAL [27] analisaram amostras de méis brasileiros e encontraram valores variando de 1,10 a $248,20 \mathrm{mg} / \mathrm{kg}$. Os autores mencionaram que os méis de países tropicais possuem alto teor de HMF, tornandose fundamental a quantificação desse componente, para a verificação da qualidade do produto.

HORN et al. [33] analisaram amostras de méis brasileiros e encontraram para a região nordeste valor médio de HMF de $113,70 \mathrm{mg} / \mathrm{kg}$, enquanto MENDES et al. [41] determinaram para méis portugueses valores de 1,70 a $94,90 \mathrm{mg} / \mathrm{kg}$.

O HMF no mel é um indicador de aquecimento, armazenamento e adulteração com açúcar invertido. WHITE JÚNIOR [64] menciona que méis de países subtropicais podem ter, naturalmente, um alto valor de HMF sem que o mel tenha sido superaquecido ou adulterado, devido a altas temperaturas. SODRÉ [53] observou valores variando de 1,50 a $136,00 \mathrm{mg} / \mathrm{kg}$, com média de $24,33 \mathrm{mg} / \mathrm{kg}$ em amostras de méis da região litoral norte da Bahia.

\subsection{8 - Índice de diastase}

O indice de diastase das 121 amostras de méis analisadas variou de 5,00 a 38,50 (escala de Gothe) com valor médio de 15,80 para méis de eucaliptos e 17,30 para méis silvestres (Tabela 2). O limite estabelecido pela legislação brasileira [12] para o índice de diastase é de no mínimo 8, desta forma 6 amostras $(4,96 \%)$ estão fora destas especificações. 
COSTA et al. [24] encontraram em amostras de méis brasileiros de diferentes origens florais valores variando de 5,90 a 66,70 (escala de Gothe) para o índice de diastase. Já ANDRADE et al. [3] observaram em méis portugueses valores variando de 13,00 a 51,10 (escala de Gothe). PERSONO-ODDO, BALDI \& ACCORTI [47] estudando a presença de enzimas em méis uniflorais italianos de diferentes origens botânicas, encontraram valores para o índice de diastase muito diferentes dentro da mesma florada.

SODRÉ [53] encontrou o índice de diastase em méis da região litoral norte do Estado da Bahia variando de 16,66 a 62,81 (escala de Gothe), com valor médio de 34,11.

As médias obtidas para índice de diastase no presente trabalho são próximas à média observada por VILHENA \& ALMEIDA-MURADIAN [60] para méis brasileiros comercializados em São Paulo.

\subsection{9 - Minerais}

Os valores de minerais obtidos nas amostras analisadas apresentaram grande variação de amostra para amostra.

O teor médio de fósforo foi de $35,82 \mathrm{mg} / \mathrm{kg}$ para méis de eucaliptos e $16,65 \mathrm{mg} / \mathrm{kg}$ para méis silvestres (Tabela 2), havendo, entretanto, uma grande variação entre os teores das amostras $(8,00$ a $155,00 \mathrm{mg} / \mathrm{kg}$ e 1,91 a $85,58 \mathrm{mg} / \mathrm{kg}$, para méis de eucaliptos e silvestres, respectivamente). A média de fósforo presente nas amostras, foi superior à média de $2,54 \mathrm{mg} / \mathrm{kg}$ encontrada por PAMPLONA [46] ao estudar amostra de méis brasileiros de eucaliptos.

O potássio foi o elemento encontrado em maior quantidade quando comparado com os demais minerais. A quantidade média foi de $1720,00 \mathrm{mg} / \mathrm{kg}(562,00 \mathrm{a}$ $3495,00 \mathrm{mg} / \mathrm{kg}$ ) e $990,85 \mathrm{mg} / \mathrm{kg}$ (95,00 a $3140,00 \mathrm{mg} / \mathrm{kg})$, respectivamente, para méis de eucaliptos e silvestres (Tabela 2).

GOMEZ et al. [32] obtiveram média de 285,60mg/kg de potássio em amostras de méis de eucaliptos da Espanha, teores esses inferiores às quantidades encontradas, nas amostras de méis, no presente experimento.

Cálcio foi o segundo elemento encontrado em maior quantidade nas amostras, com uma média de $187,70 \mathrm{mg} /$ $\mathrm{kg}(55,00$ a $301,00 \mathrm{mg} / \mathrm{kg})$ para méis de eucaliptos e $46,18 \mathrm{mg} / \mathrm{kg}(1,00$ a $202,00 \mathrm{mg} / \mathrm{kg})$ para méis silvestres (Tabela 2). GOMEZ et al. [32] encontraram os teores de $155 \mathrm{mg} / \mathrm{kg}$ de cálcio em amostras de méis de eucaliptos.

Pelos dados obtidos verifica-se que o teor de cálcio nas amostras de méis de eucaliptos é mais de três vezes superior aos valores obtidos nos méis silvestres.

A quantidade média de magnésio presente nas amostras foi de $128,74 \mathrm{mg} / \mathrm{kg}(31,00$ a $270,0 \mathrm{mg} / \mathrm{kg})$ para méis de eucaliptos e $65,45 \mathrm{mg} / \mathrm{kg}$ ( 11,00 a $286,00 \mathrm{mg} / \mathrm{kg}$ ) para méis silvestres (Tabela 2). Esse mineral foi o terceiro elemento obtido em maior quantidade nas amostras de méis de eucalipto e segundo em méis silvestres.
O enxofre foi encontrado na quantidade média de $51,82 \mathrm{mg} / \mathrm{kg}(36,00$ a $164,00 \mathrm{mg} / \mathrm{kg})$ para méis de eucaliptos e $62,47 \mathrm{mg} / \mathrm{kg}$ (1,00 a 500,00mg/kg) para méis silvestres (Tabela 2).

Os valores médios de cobre presentes nas amostras de méis de eucaliptos $0,34 \mathrm{mg} / \mathrm{kg}(0,20$ a 0,60$)$ e de silvestres $0,32 \mathrm{mg} / \mathrm{kg}(0,10$ a 2,00$)$ (Tabela 2$)$ foram inferiores aos valores obtidos por SHAVONOV e IBRISHIMOV [51], que encontraram valores de 1,81 a $8,15 \mathrm{mg} / \mathrm{kg}$ em amostras de méis da Bulgária de diferentes origens florais. GOMEZ et al. [32] obtiveram valores médios de $1,74 \mathrm{mg} / \mathrm{kg}$ de cobre em amostras de méis de eucaliptos.

Para o ferro foi encontrado o valor médio de $5,35 \mathrm{mg} / \mathrm{kg}(2,10$ a 9,00mg/kg) para as amostras de méis de eucaliptos e $10,62 \mathrm{mg} / \mathrm{kg}(3,90$ a $44,70 \mathrm{mg} / \mathrm{kg})$ para méis silvestres (Tabela 2). Esses valores foram inferiores aos obtidos por GOMEZ et al. [32].

$\mathrm{O}$ manganês esteve presente na quantidade média de $8,43 \mathrm{mg} / \mathrm{kg}(1,60$ a $16,90 \mathrm{mg} / \mathrm{kg})$ para os méis de eucaliptos e $4,46 \mathrm{mg} / \mathrm{kg}(0,20$ a $23,30 \mathrm{mg} / \mathrm{kg})$ para os méis silvestres (Tabela 2).

O zinco ocorreu na quantidade média de $2,65 \mathrm{mg} / \mathrm{kg}$ $(0,80$ a $8,70 \mathrm{mg} / \mathrm{kg})$ para os méis de eucaliptos e $5,45 \mathrm{mg} / \mathrm{kg}(1,20$ a $48,30 \mathrm{mg} / \mathrm{kg})$ para os méis silvestres (Tabela 2). O teor de zinco encontrado foi superior à quantidade de $0,09 \mathrm{mg} / \mathrm{kg}$ observado por PAMPLONA [46], ao analisar amostras de méis brasileiros de Eucalyptus.

O sódio foi encontrado na quantidade média de $71,98 \mathrm{mg} / \mathrm{kg}(23,70$ a $212,50 \mathrm{mg} / \mathrm{kg})$ para méis de eucaliptos e 49,31 mg/kg (14,95 a 202,40mg/kg) para méis silvestres (Tabela 2). GOMEZ et al. [32] verificaram a quantidade média de $77,70 \mathrm{mg} / \mathrm{kg}$ de sódio em amostras de méis de eucaliptos na Espanha.

PAMPLONA [46] estudando méis brasileiros, cita que o tipo de clima de cada região influencia no conteúdo mineral do mel, e que amostras de méis provenientes de locais com solo do tipo latossolo roxo eutrófico, apresentaram menores quantidades de ferro e manganês, do que amostras provenientes de solos formados por rochas ácidas e alcalinas.

Comparando as duas origens florais, observa-se que maiores teores de cinzas foram obtidos para méis silvestres $(0,25 \%)$ em comparação com méis de eucaliptos $(0,16 \%)$, enquanto os teores dos diversos minerais foram menores nos méis silvestres.

\section{2 - Análise de agrupamentos}

Para a análise de agrupamentos por componentes principais de 121 amostras de méis e dos 22 caracteres físico-químicos existentes na Tabela 2, foram selecionados os seguintes: proteínas, cinzas, umidade, $\mathrm{Ca}, \mathrm{S}$, $\mathrm{Cu}, \mathrm{Fe}, \mathrm{Mn}, \mathrm{Zn}, \mathrm{Na}$, acidez, índice de formol, índice de diastase, HMF, açúcares redutores, açúcares totais, sacarose aparente, sendo descartados quatro: condutividade elétrica, $\mathrm{pH}, \mathrm{K}$ e $\mathrm{Mg}$, devido à alta correlação apresentada e utilizando-se o critério proposto por JOLIFFE [38]. 
TABELA 3. Estimativas das variâncias (autovalores) e porcentagem acumulada da variância total (\%), obtidas através da análise de componentes principais considerando as 121 amostras de mel e 18 caracteres físico-químicos.

\begin{tabular}{ccc}
\hline \multirow{2}{*}{ Componentes principais } & \multicolumn{2}{c}{ Amostras de mel } \\
\cline { 2 - 3 } & Autovalores & \% acumulada \\
\hline$Y_{1}$ & 5,876 & 26,71 \\
$Y_{2}$ & 3,008 & 40,38 \\
$Y_{3}$ & 1,881 & 48,93 \\
$Y_{4}$ & 1,793 & 57,08 \\
$Y_{5}$ & 1,653 & 64,69 \\
$Y_{6}$ & 1,212 & 70,11 \\
\hline
\end{tabular}

Os resultados das estimativas de variância (autovalores) obtidos encontram-se na Tabela 3.

Pela Tabela 3 observa-se que o primeiro componente concentrou $26,71 \%$ da variância total, o segundo $40,38 \%$, o terceiro $48,93 \%$, o quarto $57,08 \%$, o quinto $64,69 \%$, e o sexto $70,11 \%$.

MARDIA, KENI \& BIBBY [40] afirmaram que se numa análise de componentes principais os dois ou três primeiros componentes acumularem uma porcentagem relativamente alta da variação total, em geral mais de $70 \%$, eles explicariam a variabilidade manifestada entre variedades avaliadas. No caso presente, observa-se que foram necessários seis componentes principais para explicar $70 \%$ da variância total disponivel

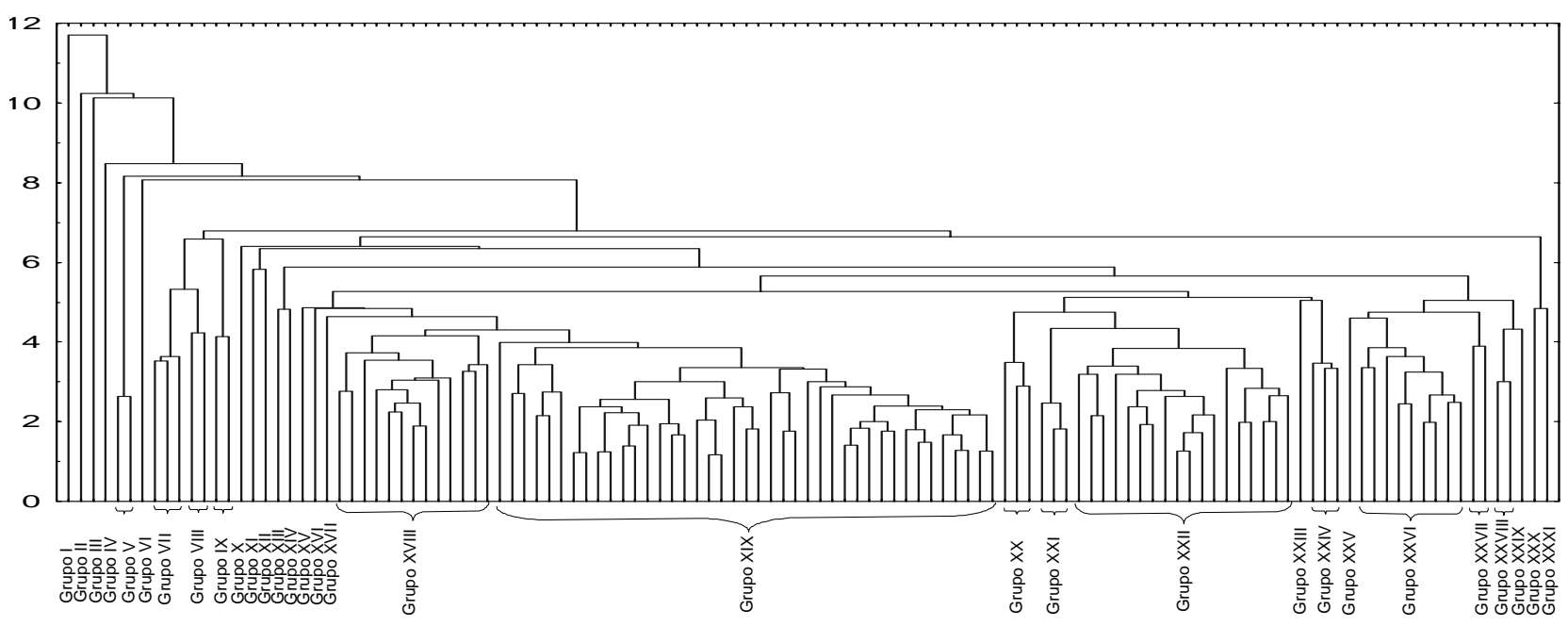

FIGURA 1. Fenograma por UPGMA e distância euclidiana média de 121 amostras de méis de Apis mellifera, do Estado de São Paulo.

\begin{tabular}{|c|c|c|c|c|c|c|c|c|c|c|c|}
\hline Grupo & $\begin{array}{c}\mathrm{N}^{\circ} \\
\text { da Amostra }\end{array}$ & Tipo de Mel & Local & Grupo & $\begin{array}{c}\mathrm{N}^{\circ} \\
\text { da Amostra }\end{array}$ & Tipo de Mel & Local & Grupo & $\begin{array}{c}\mathrm{N}^{\circ} \\
\text { da Amostra }\end{array}$ & Tipo de Mel & Local \\
\hline I & 82 & silvestre & Guareí & XIX & 12 & silvestre & Jarinu & XXI & 119 & eucalipto & Itapira \\
\hline II & 111 & eucalipto & Araraquara & XIX & 69 & silvestre & Juanópolis & XXII & 100 & eucalipto & São José do Rio Preto \\
\hline III & 6 & silvestre & Lencóois Paulista & XIX & 68 & silvestre & Itapira & XXI & 98 & eucalipto & Torrinha \\
\hline IV & 57 & silvestre & Piracicaba & XIX & 61 & silvestre & S. João da Boa Vista & XXII & 103 & eucalipto & Luís Antonio \\
\hline $\mathrm{V}$ & 23 & silvestre & São Luís do Paraitinga & XIX & 46 & silvestre & Pirassununga & XXII & 105 & eucalipto & Barretos \\
\hline $\mathrm{V}$ & 17 & silvestre & Tremembé & XIX & 91 & silvestre & Mirassol & XXII & 110 & eucalipto & Luís Antonio \\
\hline VI & 106 & eucalipto & Jaboticabal & XIX & 56 & silvestre & Colina & XXII & 121 & eucalipto & Araçatuba \\
\hline VII & 76 & silvestre & Jundiaî & XIX & 45 & silvestre & Juquitiba & XXII & 102 & eucalipto & Bebedouro \\
\hline VII & 71 & silvestre & Tietê & XIX & 47 & silvestre & Barretos & XXII & 101 & eucalipto & Altinópolis \\
\hline VII & 66 & silvestre & Vista Alegre do Alto & XIX & 65 & silvestre & Vargen Grande do Sul & XXII & 96 & eucalipto & Lençóis Paulista \\
\hline VIII & 87 & silvestre & Lins & XIX & 39 & silvestre & $\begin{array}{l}\text { Altinopolis } \\
\text { Sul }\end{array}$ & XXII & 109 & eucalipto & Avaré \\
\hline VII & 32 & silvestre & Serra Negra & XIX & 92 & silvestre & Estrela d'Oeste & XXII & 108 & eucalipto & Avaré \\
\hline IX & 11 & silvestre & Jundiai & XIX & 93 & silvestre & Estrela d'Oeste & XXII & 99 & eucalipto & Lençóis Paulista \\
\hline IX & 10 & silvestre & Torrinha & XIX & 85 & silvestre & Valentim Gentil & XXII & 95 & eucalipto & Anhembi \\
\hline$x$ & 90 & silvestre & Brotas & XIX & 67 & silvestre & Mirandópolis & XXII & 107 & eucalipto & Botucatu \\
\hline$\hat{\mathrm{X}} \mathrm{I}$ & 70 & silvestre & Paranapanema & XIX & 48 & silvestre & Itapeva & XXII & 114 & eucalipto & Brotas \\
\hline XII & 3 & silvestre & Guarujá & XIX & 28 & silvestre & Bananal & XXII & 104 & eucalipto & Itapetininga \\
\hline XIII & 29 & silvestre & Serra Negra & XVIII & 58 & silvestre & Igarapava & XXII & 117 & eucalipto & Brotas \\
\hline XIV & 27 & silvestre & Santo Antonio do Pinhal & XVIII & 30 & silvestre & Serra Negra & XXII & 115 & eucalipto & Ipiúna \\
\hline XV & 37 & silvestre & Itapirapuã Paulista & XVIII & 25 & silvestre & Lagoinha & XXII & 5 & silvestre & Rio Claro \\
\hline XVI & 60 & silvestre & Ituverava & $\begin{array}{l}\text { XVIII } \\
\text { XV } 19\end{array}$ & 9 & silvestre & Torrinha & XXIII & 49 & silvestre & Monte Alegre do Sul \\
\hline XVII & 24 & $\begin{array}{l}\text { silvestre } \\
\text { siles }\end{array}$ & São Luís do Paraitinga & XVIII & 52 & $\begin{array}{l}\text { silvestre } \\
\text { siles }\end{array}$ & São Roque & XXIV & 38 & silvestre & E.Santo do Pinhal \\
\hline XVIII & 63 & silvestre & Guarani d'Oeste & XVIII & 40 & silvestre & Itaberá & XXIV & 33 & silvestre & Piracaia \\
\hline XVIII & 54 & silvestre & Guareí & XVIII & 75 & silvestre & Araçatuba & XXIV & 4 & silvestre & Bofete \\
\hline XVIII & 43 & silvestre & Itapetininga & XVIII & 41 & silvestre & Boa Esperanca do Sul & $\mathrm{XXV}$ & 86 & silvestre & Gália \\
\hline XVIII & 73 & silvestre & Assis & XVIII & 36 & silvestre & Capão Bonito & XXVI & 83 & silvestre & Junqueirópolis \\
\hline XVIII & 72 & silvestre & Andradina & XVIII & 77 & silvestre & São Paulo & XXVI & 80 & silvestre & Auriflama \\
\hline XVIII & 53 & silvestre & $\begin{array}{l}\text { Oriente } \\
\text { Ona }\end{array}$ & XVIII & 35 & silvestre & São José do Rio Preto & XXVI & 94 & silvestre & Presidente Prudente \\
\hline XVIII & 64 & silvestre & Ilha Solteira & XVIII & 34 & silvestre & São José do Rio Preto & XXVI & 88 & silvestre & Sabino \\
\hline XVIII & 42 & silvestre & Castilho & XVIII & 59 & silvestre & Monteiro Lobato & XXVI & 79 & silvestre & Auriflama \\
\hline XVIII & 31 & silvestre & Serra Negra & XVIII & 26 & silvestre & Redenção da Serra & XXVI & 89 & silvestre & Sabino \\
\hline XVIII & 18 & silvestre & Redencão da Serra & XVIII & 21 & silvestre & Cacapava & XXVI & 78 & silvestre & Aracatuba \\
\hline XVIII & 44 & silvestre & Santa Cruz do Rio Preto & $\begin{array}{l}\text { XVIII } \\
\text { XII }\end{array}$ & 22 & silvestre & Paraibuna & XXVI & 84 & silvestre & Junqueirópolis \\
\hline XVIII & 19 & silvestre & Taubaté & XVIII & 16 & silvestre & Piracuama & XXVI & 62 & silvestre & Santa Fé do Sul \\
\hline $\begin{array}{l}\text { XVIII } \\
\text { XVIII }\end{array}$ & 13 & $\begin{array}{l}\text { Silvestre } \\
\text { silvestre }\end{array}$ & $\begin{array}{l}\text { Taubate } \\
\text { Jundiaí }\end{array}$ & XVIII & 8 & silvestre & São Pedro & $\begin{array}{l}\text { XXVII } \\
\text { XXV }\end{array}$ & 81 & silvestre & Sete Barros \\
\hline XIX & 55 & silvestre & Capela do Alto & XVIII & 7 & silvestre & Carumbataí & XXVII & 20 & $\begin{array}{l}\text { silvestre } \\
\text { siles }\end{array}$ & Ubatuba \\
\hline XIX & 112 & eucalipto & Monteiro Lobato & $\mathrm{xX}$ & 118 & eucalipto & Cacapava & XXVIII & I 51 & silvestre & São Roque \\
\hline XIX & 15 & silvestre & Cunha & $\mathrm{XX}$ & 116 & eucalipto & Itapira & XXVIII & I 50 & silvestre & São Roque \\
\hline XIX & 74 & silvestre & São Carlos & $x x$ & 113 & eucalipto & Vargem Grande do Sul & XXIX & 2 & silvestre & Itanhaém \\
\hline XIX & 14 & silvestre & Votorantin & XXI & 120 & eucalipto & São Paulo & $\mathrm{XXX}$ & 97 & eucalipto & Rio Claro \\
\hline & & & & & & & & XXXI & 1 & silvestre & Serra Negra \\
\hline
\end{tabular}


entre os caracteres físico-químicos. Assim, constatase uma considerável dispersão da variância no material estudado, razão pela qual optou-se pela análise de Cluster.

Os dados da análise de Cluster podem ser observados no fenograma da Figura 1.

Pela Figura 1 observa-se que foram formados 31 grupos, os quais podem ser identificados a seguir: no grupo I encontra-se uma amostra (no 82); no grupo II encontrase uma amostra (no 111); no grupo III encontra-se uma amostra (no 6); no grupo IV, uma amostra (no 57): no grupo V, duas amostras ( $\mathrm{n}^{\circ} 23,17$ ); no grupo VI, uma amostra (no 106); no grupo VII, três amostras (no 76, 71, 66); no grupo VIII, duas amostras (no 87,32 ); no grupo IX, duas amostras (no 11,10$)$; no grupo X, uma amostra (no 90); no grupo XI, uma amostra ( ${ }^{\circ}$ 70); no grupo XII, uma amostra (no 3 ); no grupo XIII, uma amostra (no 29$)$ : no grupo XIV, uma amostra (no 27); no grupo XV, uma amostra (n⿳o 37); no grupo XVI, uma amostra ( $n^{\circ} 60$ ); no grupo XVII, uma amostra (no 24); no grupo XVIII, treze amostras (no $63,54,43,73,72,53,64,42,31,18,44,19,13)$; no grupo XIX, quarenta e uma amostras (no $55,112,15$, $74,14,12,69,68,61,46,91,56,45,47,65,39,92,93,85$, 67, 48, 28, 58, 30, 25, 9, 52, 40, 75, 41, 36, 77, 35, 34, 59, $26,21,22,16,8,7$ ); no grupo XX, três amostras (no 118 , $116,113)$; no grupo XXI, três amostras (no $120,119,98)$; no grupo XXII, dezoito amostras (no 103, 105, 100, 110, $121,102,101,96,109,108,99,95,107,114,104,117$, $115,5)$; no grupo XXIII, uma amostra (no 49); no grupo XXIV, três amostras (no $38,33,4)$; no grupo XXV, uma amostra (no 86); no grupo XXVI, nove amostras (no 83, 80, 94, 88, 79, 89, 78, 84, 62); no grupo XXVII, duas amostras (no 81, 20); no grupo XXVIII, duas amostras ( $\mathrm{n}^{\circ}$ 51, 50); no grupo XXIX, uma amostra (no 2); no grupo XXX, uma amostra (no 97); no grupo XXXI, uma amostra (no 1).

Adotou-se como critério para definição dos grupos o gráfico das distâncias de ligação nos sucessivos passos da análise de agrupamento. Este método baseia-se na identificação de um platô no sentido vertical, o que significa que muitos grupos foram formados na mesma distância de ligação. Esta distância pode ser um ponto ótimo de corte no dendrograma determinando o número de grupos formados. No presente trabalho o ponto ótimo para corte escolhido foi 4,3, representado pela linha tracejada na Figura 1. O ponto ótimo para o corte foi baseado na identificação de um platô no sentido vertical, o que significa que muitos grupos foram formados na mesma distância de ligação, sendo esta distância o ponto ótimo de corte para o fenograma, determinando o número de grupos formados (Figura 2)

Observa-se que os méis de eucaliptos formaram dois grupos ficando apenas quatro amostras (amostras 97, 106, 111, 112) (legenda da Figura 1), declaradas como de eucaliptos, fora destes grupos. Pela análise polínica destas quatro amostras verificou-se que elas continham pólen de eucaliptos, mas, uma quantidade variável de outros pólens, podendo, portanto, ser classificados como méis poliflorais. As demais 23 amostras declaradas como de eucalipto foram confirmadas pela análise polínica. Por outro lado os méis declarados pelos apicultores como silvestres, o que foi confirmado pela análise polínica, formaram diversos grupos devido a grande variedade de tipos polínicos.

Pela análise dos componentes principais, pode-se verificar que os caracteres que mais influenciaram no agrupamentos das amostras de méis foram condutividade elétrica e quantidade de $\mathrm{K}$ no eixo $\mathrm{X}$ e índice de formol e umidade no eixo Y.

Segundo BOGDANOV [10] a condutividade elétrica é considerada um bom critério para indicar a origem floral do mel pois é influenciada por ácidos e pelo conteúdo de cinzas. Méis de diferentes origens florais têm valores diferentes de condutividade elétrica.

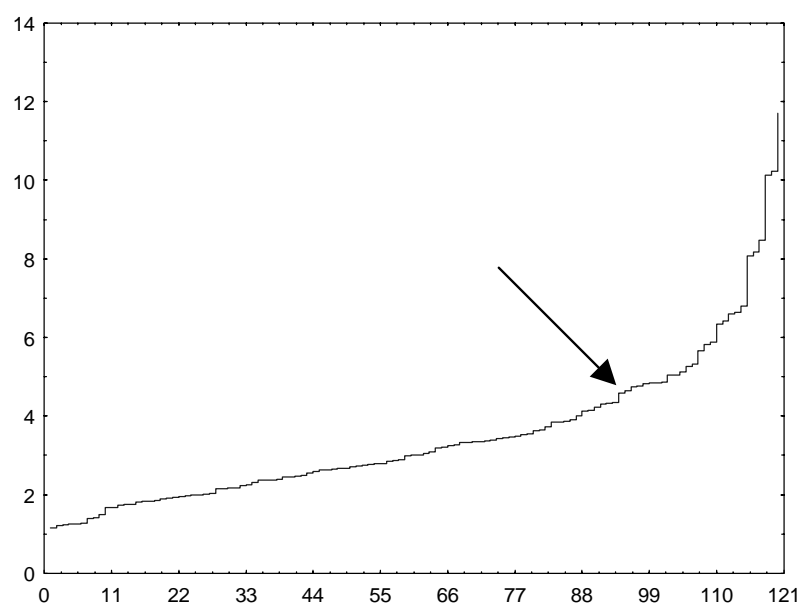

FIGURA 2. Gráfico das distâncias de ligação nos sucessivos passos de agrupamento utilizando a distância euclidiana média e o método do UPGMA. A seta está indicando a distância de corte no fenograma para a definição dos grupos na Figura 1.

\section{4 - CONCLUSÕES}

Dentre as 121 amostras de méis do Estado de São Paulo analisadas as de eucaliptos e as silvestres formam grupos distintos quanto aos caracteres físico-químicos, o que confirma que a origem floral interfere decisivamente nas características dos méis.

Os valores médios dos parâmetros analisados e que constam da legislação vigente, encontram-se dentro dos limites estabelecidos, com exceção da umidade em $36,40 \%$ das amostras de méis de eucaliptos.

\section{5 - REFERÊNCIAS BIBLIOGRÁFICAS}

[1] AGANIN, A.F. Electrical condutivity of several unifloral honeys. Trudy Saratovskogo Zootekhnicheskogo Instituta, v. 21 , p. 137-144, 1971. Resumo em Apicultural Abstracts, v. 25, n. 1, p. 144, 1973.

[2] AMARAL, A.D.; IMPERATRIZ-FONSECA, V.L.; KLEINERT-GIOVANNINI, A.K.; AZOUBEL, M.L. Estudo comparativo entre amostras de méis com origem flo- 
ral determinada; análise do teor de proteínas, lipídios e carboidratos totais. In: CONGRESSO BRASILEIRO DE APICULTURA, 7., Salvador, 1986. Anais. Salvador: Confederação Brasileira de Apicultura, 1986. p.60.

[3] ANDRADE, P.B.; AMARAL, M.T.; ISABEL, P.; CAMARGO, J.C.M.F.; SEABRA, R.M.; CUNHA, A.P. Physicochemical attributes and pollen spectrum of Portuguese heather honeys. Food Chemistry, v. 66, n. 4, p. 503-510, 1999.

[4] ATAGo Co. Refratômetro para mel. Abelhas, v. 31, n. 362/363, p. 9, 11-12, 41,44, 1988. Resumo em CAB, 1987-89.

[5] AZEREDO, M.A.A.; AZEREDO, L. da C. Características físico-químicas dos méis do município de São FidélisRJ. Ciênc. Tecnol. Aliment., v. 19, n. 1, p. 3-7, 1999.

[6] BALDI-CORONEL, B.; DALL'OGLLIO, A.M.; LEZCANO, S. Caracterización físico químico de las mieles de la Provincia de Entre Rios. Alimentación Latinoamericana, n. 39, p. 39-44, 1993.

[7] BARTH, M. O. O pólen no mel brasileiro. Rio de Janeiro: Luxor, 1989. 152p.

[8] BATH, P.K.; SINGH, N. A comparison between Helianthus annuus and Eucalyptus lanceolatus honeys. Food Chemistry, v. 67, n. 4, p. 389-397, 1999.

[9] BOLETIN OFICIAL ESPAÑOL (B.O.E.) Orden de 12 de junio de 1986, de la Presidencia del Gobierno por la que se aprueban los métodos oficiales de analisis para la miel. B.O.E., Madrid, 18 junio de 1986, n. 145, s.n.p.

[10] BOGDANOV, S. Honey quality and international regulatory standards: review by the international honey commission. Bee World, v. 80, n. 2, p. 61-69, 1999.

[11] BOGDANOV, S.; MARTIN, P.; LULLMANN, C. Harmonized methods of the european honey commission. Apidologie, Extra. Issue, p. 1-59, 1997.

[12] BRASIL. Ministério da Agricultura. Instrução normativa 11 , de 20 de outubro de 2000. Regulamento técnico de identidade e qualidade do mel. Diário Oficial, Brasília, 20 de outubro de 2000, Seção 1, p. 16-17.

[13] BUSSAB. W.O.; ANDRADE, D.F.; MYAZAKY, E.S. Introdução a análise de agrupamento. In: SIMPÓSIO NACIONAL DE PROBABILIDADE E ESTATÍSTICA, 9., São Paulo, 1990. Anais. São Paulo: IME/USP, 1990. $105 p$.

[14] CAMPOS, G. Melato no mel e sua determinação através de diferentes metodologias. Belo Horizonte. 1998. 178p. Tese (Doutorado) - Escola de Veterinária, Universidade Federal de Minas Gerais.

[15] CAMPUS, R.; MADAU, G.; SOLINAS, B. Composition of Sardinian honeys: content of nitrogenous and polyphenolic substances. Tecnologie Alimentari, v. 6, n. 10, p. 10-15, 1983. Resumo em Apicultural Abstracts, v. 37, n. 3, p. 245, 1986.

[16] CANO, C.B.; FELSNER, M.L.; MATTOS, J.R.; BRUNS, R.E.; WATANABE, H.M.; MURADIAN, L.B.A. Comparison of methods for determining moisture content of citrus and eucaplyptus brazilian honeys by refractometry. Journal of Food Composition Analysis, v. 14, p. 101-109, 2001.

[17] CANO, C.B.; ZAMBONI, C.Q.; ALVES, H.I; SPITERI, N.; ATUI, M.B.; SANTOS, M.C. dos; JORGE, L.I.F.; PEREIRA, U.; RODRIGUES, R.M.M. Mel: fraudes e condições sanitárias. Revista do Instituto Adolfo Lutz, v. 52, p. 1-4, 1992.

[18] CARVALHO, C.A.L. de; MARCHINI, L.C.; SODRÉ, G. da S; ALVES, R.M. de O.; PASSOS, L. Análises de amostras de méis provenientes do recôncavo da Bahia. In: ENCONTRO SOBRE ABELHAS, 4., Ribeirão Preto, 2000. Anais. Ribeirão Preto: Faculdade de Filosofia Ciências e Letras, 2000. p.352.

[19] CARVALHO, C.A.L. de; MARCHINI, L.C.; TEIXEIRA, G.M.; OLIVEIRA, P.C. F. de; RUBIA, V.R. Características físico-químicas de amostras de méis da Bahia. In: CONGRESSO BRASILEIRO DE APICULTURA, 12., Salvador, 1998. Anais. Salvador: Confederação Brasileira de Apicultura, 1998, p. 200.

[20] CIRILli, G.; PAPAGHEORGHeU, A.; SAVIGNi, G. Chemical and nutritional characteristics of honey. Industrie Alimentari, Pinerolo, v. 12, n. 4, p. 74-76, 1973. Resumo em Apicultural Abstracts, v. 27, n. 2, p. 117-118, 1976.

[21] COPERSUCAR (Cooperativa de Produtores de Cana, Açúcar e Álcool do Estado de São Paulo). Métodos Anaíticos. In: COPESUCAR. Manual de Controle Químico da Fabricação de Açúcar. Piracicaba:Copersucar, 1987. p. $1-51$

[22] CORNEJO, L.G. Tecnologia de miel. In: SEEMANN, P.; NEIRA, M. (ed). Tecnologia de la produccion apicola. Valdivia: Universidad Austral de Chile, Facultad de Ciencias Agrarias, 1988. p.145-71.

[23] CORNEJO, L.G.; TOMASEVICH, R. Estudio sumario de la calidade de las mieles de algunas zonas del Estado de Rio Grande do Sul - Brasil. In: CONGRESSO BRASILEIRO DE APICULTURA, 1., Florionópolis, 1970. Anais. Florionópolis: Confederação Brasileira de Apicultura, 1970, p.241-245.

[24] COSTA, L.S.M.; ALBUQUERQUE, M.L.S.; TURGO, L.C.; QUINTEIRO, L.; BARTH, O.M.; RIBEIRO, M.; MARIA, C.A.B. Determination of non-volatile compounds of different botanical origin brazilian honeys. Food Chemistry, v. 65, p. 347-352, 1999.

[25] CRANE, E. Bees and beekeeping-science, practice and world resources. London: Neinemann Newnes, 1990. $614 \mathrm{p}$.

[26] CRANE, E. Honey: a comprehensive survey. London: Heinemann, 1975. 608p.

[27] DAYRELL, I.O.; VITAL, N.C. Comparação entre dois métodos oficiais para determinação de hidroximetilfurfural (HMF) em mel brasileiro. Ciênc. Tecnol. Aliment., v. 1, n. 1, p. 137-141, 1991.

[28] ERDTMAn, G. Pollen morphology and plant taxonomy - Angiosperms. Stockholm: Almqvist \& Wiksell, 1952. 539p.

[29] FLECHTMANN, C.H.W.; CALDAS FILHO, C.F.; AMARAL, E.; ARZOLLA, J.D.P. Análise de méis do estado de São Paulo. Boletim de Indústria Animal, v. 21, p. 65-73, 1963.

[30] FRÍAS, I.; HARDISSON, A. Estudio de los parámetros analíticos de interés en la miel. II. Azúcares, cenizas y contenido mineral y color. Alimentaria, v. 28, n. 235, p. 41-43, 1992.

[31] GAMERO, A.M.; CORNEJO, L.G.; TOMASEVICH, R. Tipification de mieles de abejas de la provincia de Buenos Aires. In: CONGRESSO BRASILEIRO DE APICULTURA, 1., Florionópolis, 1970. Anais. Florianópolis: Confederação Brasileira de Apicultura, 1970, p.272-289.

[32] GOMEZ, M.E.M.; HERNANDEZ, E.G.; GOMEZ, J.Y.M.; MARIN, J.L.M. Physicochemical analysis of Spanish commercial Eucaliptus honeys. Journal of Apicultural Research, v. 32, n. 3/4, p. 121-126, 1993.

[33] HORN, H.; DURÁN, J.E.T.; CORTOPASSI-LAURINO, 
M.; ISSA, M.R.C.; TOLEDO, V.A.A. de; BASTOS, E.; SOARES, A.E.E. Méis brasileiros: resultados de análise físico-químicas e palinológicas. In: CONGRESSO BRASILEIRO DE APICULTURA, 11., Teresina, 1996. Anais. Teresina: CBA, 1996, p. 403-429.

[34] HOWER, F.N. Plantas melíferas. Barcelona: Reverté, 1953. 35p.

[35] IMPERATRIZ-FONSECA, V.L.; GIOVANNINI, A. GUIBU, L.S.; AZOUBEL, M.L.; AMARAL, A.D. Preliminary study of Brasilian honeys. In: INTERNATIONAL APICULTURAL CONGRESSO, 30., Nogoya, 1987. Proceedings. Nagoya: APIMONDIA, 1987. p. 312.

[36] INTERNATIONAL TRADE FORUM Upswing in the honey market. International Trade Forum, Geneva, v. 13, n. 3, p. 21-31, 1977. Resumo em Apicultural Abstracts, v. 30, n. 3, p. 214, 1979.

[37] IWAMA, S.; MELHEM, T. S. The pollen spectrum of the honey of Tetragonisca angustula angustula Latrelle (Apidae, Meliponinae). Apidologie, v. 10, n. 3, p. 275295, 1979.

[38] JOLIFFE, I.T. Discarding variables in a principal component analysis. I Real date. Applied Statistics, v. 21, n. 1, p. 21-31, 1973.

[39] LASCEVE, G.; GONNET, M. Analyse por radioactivation du contenu mineral d'un miel. Possibilité de preciser son origine. Apidologie, v. 5, n. 3, p. 201-223, 1974.

[40] MARDIA, L.V.; KENI, J.T.; BIBBY, J.M. Multivariate analysis. London: Academic Press, 1979. 521p.

[41] MENDES, E.; PROENCA, E.B.; FERREIRA, I.M.P.L.V.O.; FERREIRA, M.A. Quality evaluation of portuguese honey. Carbohydrate Polymers, v. 37, n. 3, p. 219-223, 1998.

[42] MORAES, R.M. de; MANTOVANI, D.M.B. Composição química de méis de diferentes fontes florais. In: CONGRESSO BRASILEIRO DE APICULTURA, 7., Salvador, 1986. Programa e resumo. Salvador: Confederação Brasileira de Apicultura, 1986. p.58.

[43] MORAES, R.M. de; TEIXEIRA, E.W. Análise de mel (Manual técnico). Pindamonhangaba:sn, 1998. 41p.

[44] MORRISON, D. F. Multivariate statistical methods. 2. ed. Tokyo: Mc Graw-Hill, 1981. 415p.

[45] ORTIZ, V.A. The ash content of 69 honey samples from La Alcarria and neighbouring areas, collected in the period 1985-87. Cuadernos de Apicultura, n. 5, p. 8-9, 1988. Resumo em Apicultural Abstracts, v. 40, n. 4, p.360, 1989.

[46] PAMPLONA, B.C. Exame dos elementos químicos inorgânicos encontrados em méis brasileiros de Apis mellifera e suas relações físico-biológicas. São Paulo. 1989. 131p. Dissertação (Mestrado) - Instituto de Biociências, Universidade de São Paulo.

[47] PERSANO-ODdO, P.L.; BALDI, E.; ACCORTI, M. Diastatic activity in some unifloral honey. Apidologie, v. 21, p. 17-24. 1990.

[48] PREGNolato, W.; PREGNOlato, N.P. (Coord). Normas analíticas do Instituto Adolfo Lutz. 3. ed. São Paulo: Instituto Adolfo Lutz, 1985. v. 1: Métodos químicos e físicos para análise de alimentos. 533p.

[49] SALINAS, F.; ESOINOSA-MANSILLA, A.; BERZASVEVADO, J. J. Flow-injection determination of HMF in honey by Winkler method. Fresenius Journal of Analytical Chemistry, v. 340, n. 4, p. 250-252, 1991.
[50] SEEMANN, P.; NEIRA, M. Tecnología de la producción apícola. Valdivia: Universidad Austral de Chile/ Facultad de Ciencias Agrarias Empaste, 1988. 202p.

[51] SHAVONOV, M.; IBRISHIMOV, N. Assessment of trace elements in the environment on the basis of honey foraging activity. Acta Microbiologica, Virologica et Immunologica. v. 2, p. 105-108, 1975. Resumo em Apicultural Abstracts, v. 28, n. 4, p. 276, 1977.

[52] SIMAL, J.; HUIDOBRO, J. Parámetros de calidade de la miel III. Acidez ( $\mathrm{pH}$, libre, lactónica \& total) e índice de formol. Offarm, v. 3 n. 9, p. 532, 1984.

[53] SODRÉ, G. da S. Características físico-químicas e análises polínicas de amostras de méis de Apis mellifera L., 1758 (HYMENOPTERA: APIDAE) da região litoral norte do Estado da Bahia. Piracicaba, SP. 2000. 83p. Dissertação (Mestrado) - Escola Superior de Agricultura "Luíz de Queiroz", Universidade de São Paulo.

[54] SPORNS, P.; PLHAK, L.; FRIEDRICH, J. Alberta honey composition. Food Research International. v. 25, n. 2, p. 93-100. 1992.

[55] STEFANINI, R. Variability and analysis of Italian honeys. Apiacta, v. 19, n. 4, p. 109-114, 1984.

[56] TEMIZ, A. I. Composition and characteristics of honeys from the Izmir region, and effects of different storage methods. Ege Bolge Zirai Arastirma Enstitusu Yayinlari, v. 31, n. 11, p. 113, 1983. Resumo em CAB Abstracts on CD-Rom, 1984-86.

[57] VANSELL, G. H.; FREEBORN, S. B. Preliminary report on the investigations of the source of diastase in honey. Journal of Economic Entomology, v. 22, p. 922-926, 1929.

[58] Vermeulen, L.; PELERENTS, C. Suiker, fosfor en ijzerghalte van Belgische Honing. Medelingen Landbouwhogeschool Gent, v. 30, n. 2, p. 527-541, 1965.

[59] VIDAL, R.; FREGOSI, E. V. de. Me1: características, análises físico-químicas, adulterações e transformações. Barretos: Instituto Tecnológico Científico "Roberto Rios", 1984. 95p.

[60] VILHENA, F.; ALMEIDA-MURADIAN, L. B. Análises físico-químicas de méis de São Paulo. Mensagem Doce, v. 53, p. 17-19, 1999.

[61] VIT-OLIVIER, P. Physical-chemical characteristics of commercial honeys from Venezuela. In: AUSTRALIAN AND INTERNATIONAL BEEKEEPING CONGRESS, 2., Queensland, 1988. Proceedings. Queensland: International Colour Prodictions, Pty. Ltd., 1988. p. 227228. Resumo em Apicultural Abstracts, v. 42, n. 2, p. $168,1991$.

[62] WHITE JUNIOR, J. R. Honey. In: GROUT, R. A. The hive and honeybee. Hamilton: Dadant \& Sons, 1976. p. 491-530.

[63] WHITE JUNIOR, J. W. Methods for determinung carbohydrates, hydroxymetilhyfurfural and proline in honey: Collaborative study. Journal of the Association of Official Analytical Chemists, v. 62, n. 3, p. 515$526,1979$.

[64] WHITE JÚNIOR, J. W. Quality evaluation of honey: role of and diastase assays. American Bee Journal, v. 132, n. 12, p. 792-794, 1992.

[65] WHITE JÚNIOR, J. W. Honey. Advances in Food Research, v. 22. p. 287-374, 1978. 